\title{
Note
}

\section{Solubility of D-Galactose, D-Talose, and D-Tagatose in Aqueous Ethanol at Low Temperature}

\author{
Da-Ming Gao, Takashi Kobayashi and Shuji AdACHI \\ Division of Food Science and Biotechnology, Graduate School of Agriculture, Kyoto University, Sakyo-ku, Kyoto 606-8502, \\ Japan
}

Received June 14, 2015 ; Accepted August 20, 2015

The solubility of D-galactose, D-tagatose, and D-talose in aqueous ethanol $(20-80 \mathrm{wt} \%)$ was measured in the temperature range of $-30^{\circ} \mathrm{C}$ to $20^{\circ} \mathrm{C}$. The solubility of each sugar decreased upon increasing the ethanol concentration and decreasing the temperature. The solubility of D-talose and D-tagatose was higher than that of D-galactose under all conditions. The temperature dependence of the solubility of each hexose can be expressed by the van't Hoff equation. The dissolution enthalpy of each hexose decreased upon increasing the ethanol concentration. The dissolution enthalpy of D-talose was higher than that of D-galactose and D-tagatose.

Keywords: D-galactose, D-talose, D-tagatose, solubility, aqueous ethanol

\section{Introduction}

D-Tagatose and D-talose, both of which are rare sugars, have many important uses such as food additives to improve health and extend life span (Levin et al., 1995; Lu et al., 2008) and raw materials to synthesize value-added products (Baer, 1962; Yu and Chung, 1968). As a result, many methods such as alkali-, metalcatalyzed, and enzymatic isomerization have been developed to produce D-tagatose and D-talose (Khadem et al., 1989; Yamauchi et al., 1990; Lim et al., 2007; Beerens et al., 2012). Among these methods, the alkali-catalyzed isomerization of D-galactose is promising for use in the food industry for reasons of food safety, pretreatment, and postpurification, although many byproducts are produced through side reactions.

We previously reported that high yields of D-tagatose can be produced by treating D-galactose with subcritical aqueous ethanol, along with the production of a small amount of D-talose (Gao et al., 2015). This method is also considered promising for the production of D-tagatose from D-galactose because of its high productivity and short reaction time. This method produces unreacted substrates, desired products, and some byproducts in the reaction effluent.
Therefore, to reduce the load on the separation process, decreasing the temperature or increasing the ethanol concentration for precipitating desirable or undesirable components before chromatographic separation is considered a possible solution as the first step in the purification process.

In this study, we measured the solubility of D-galactose, $\mathrm{D}$-tagatose, and D-talose in aqueous ethanol in the temperature range of $-30^{\circ} \mathrm{C}$ to $20^{\circ} \mathrm{C}$.

\section{Materials and Methods}

Materials Ethanol, D-galactose, D-tagatose, and D-talose were purchased from Wako Pure Chemical Industries (Osaka, Japan). In this study, the solubility of only D-enantiomers was investigated, and therefore, the prefix, D-, of all hexoses is omitted hereafter.

Solubility measurement The solubility of galactose, tagatose, and talose in aqueous ethanol $(20-80 \mathrm{wt} \%)$ was measured in the temperature range of $-30^{\circ} \mathrm{C}$ to $20^{\circ} \mathrm{C}$. The temperature was controlled using an FP89-HL ultralow refrigerated-heating circulator (Julabo Japan, Osaka, Japan). A solution of galactose, tagatose, or talose was prepared in a vial by adding an excess 


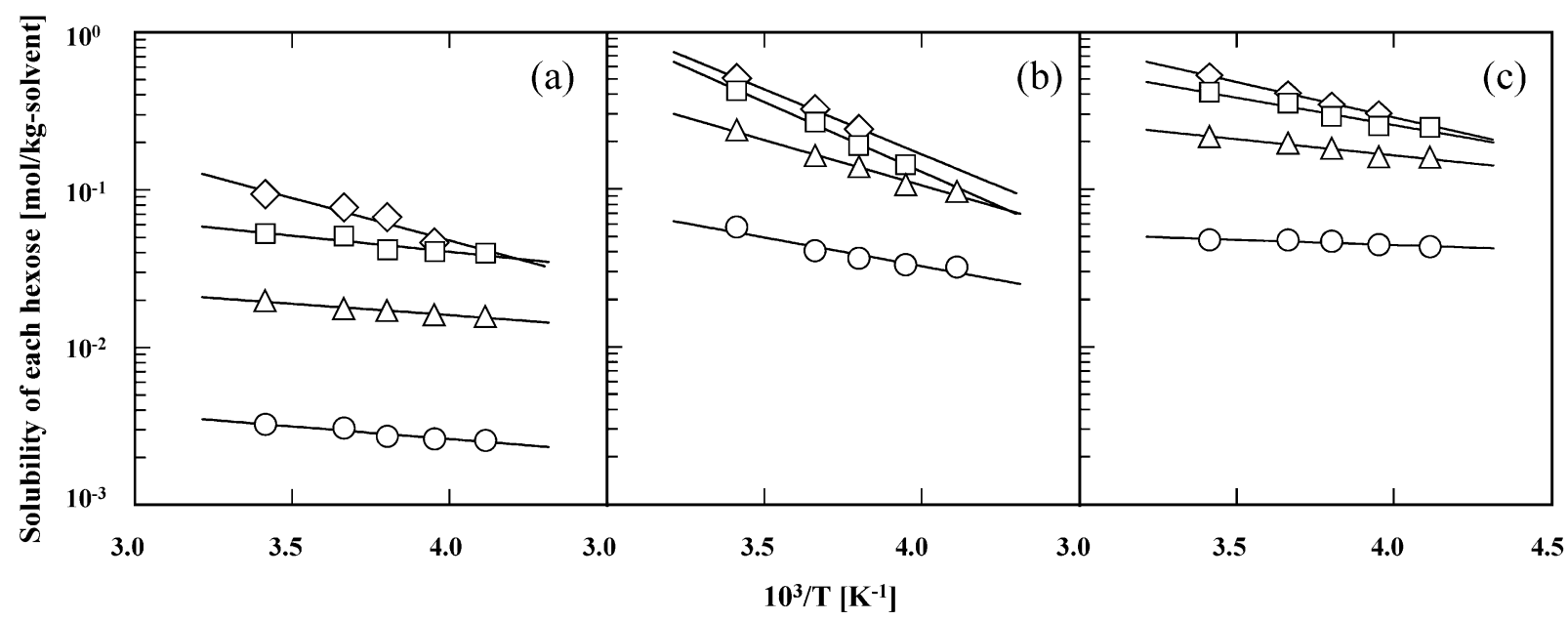

Fig. 1. Temperature dependence of solubility of (a) galactose, (b) talose, and (c) tagatose in $(\diamond) 20 \mathrm{wt} \%,(\square) 40 \%,(\triangle) 60 \%$, and $(\bigcirc) 80 \%$ ethanol.

Table 1. Dissolution enthalpies of galactose, talose, and tagatose, $\Delta H_{i}$, in aqueous ethanol.

\begin{tabular}{cccc}
\hline $\begin{array}{c}\text { Ethanol concentration } \\
{[\mathrm{wt} \%]}\end{array}$ & Galactose & Talose & Tagatose \\
\cline { 2 - 4 } & 10 & 16 & 8.6 \\
20 & 3.9 & 17 & 6.7 \\
60 & 3.1 & 11 & 3.9 \\
80 & 3.0 & 6.9 & 1.3 \\
\hline
\end{tabular}

amount of hexose to aqueous ethanol. The solution was kept at the measuring temperature for $24 \mathrm{~h}$ to ensure equilibrium solubility.

The supernatant of the hexose solution was diluted to a detectable concentration for HPLC quantification with aqueous ethanol. The HPLC system consisted of an LC-10AD VP HPLC pump (Shimadzu, Kyoto, Japan), RI-101 refractometer (Showa Denko, Tokyo, Japan), and a Cosmosil Sugar-D column (4.6 mm I.D. $\times 250 \mathrm{~mm}$ length; Nacalai Tesque, Kyoto, Japan). The column was maintained at $30^{\circ} \mathrm{C}$ in a CTO-10A VP column oven (Shimadzu). A water-acetonitrile (20:80, v/v) mixture was used as the eluent at a flow rate of $1.0 \mathrm{~mL} / \mathrm{min}$.

\section{Results and Discussion}

Figures 1(a), (b), and (c) show the solubility of galactose, talose, and tagatose in $20-80 \mathrm{wt} \%$ aqueous ethanol at various temperatures according to the van't Hoff equation:

$$
\frac{\mathrm{d} \ln S_{i}}{\mathrm{~d}(1 / T)}=-\frac{\Delta H_{i}}{R}
$$

where $S_{i}$ is the solubility of hexose $i ; \Delta H_{i}$, the dissolution enthalpy of hexose $i$; $R$, the gas constant; $T$, the absolute temperature; and subscript $i$, galactose, talose, or tagatose. The solubility of all hexoses decreased upon increasing the ethanol concentration and decreasing the temperature. For example, at $20^{\circ} \mathrm{C}$, the solubility of galactose, talose, and tagatose decreased from 0.093 to $0.003,0.502$ to $0.057,0.527$ to $0.048 \mathrm{~mol} / \mathrm{kg}$-solvent, respectively, upon changing the solvent from $20 \mathrm{wt} \%$ aqueous ethanol to $80 \mathrm{wt} \%$ aqueous ethanol. The solubility of galactose and tagatose in $40 \mathrm{wt} \%$ aqueous ethanol both decreased by $\sim 40 \%$ upon decreasing the temperature from $20^{\circ} \mathrm{C}$ to $-30^{\circ} \mathrm{C}$, and by $\sim 66 \%$ from $20^{\circ} \mathrm{C}$ to $-20^{\circ} \mathrm{C}$ for talose. Further, the hexose solubility was more sensitive to temperature change at lower ethanol concentration (Alves et al., 2007; Flood et al., 1996), indicating that the solvent with low ethanol concentration was favorable for obtaining a solution with high tagatose content by hexose crystallization, which would be similar to the separation of fructose and glucose by freezing crystallization (Silva et al., 2010). The solubility of talose and tagatose was much higher than that of galactose under all measured conditions. Hexoses exist as equilibrium mixtures of pyranose and furanose with $\alpha$ - or $\beta$-configurations. Therefore, the temperature dependence of the saccharide solubility cannot be simply explained. However, it was reported that the solubility of mannose and fructose in aqueous ethanol was higher than that of glucose at the same temperature and ethanol concentration (Hudson and Yanovsky, 1917). Because talose, tagatose, and galactose were the C-4 epimers of mannose, fructose, and glucose, respectively, this tendency may be also applicable to the difference in solubility of talose, tagatose, and galactose. The difference in solubility increased with increasing ethanol concentration. In particular, the solubility of talose was the most sensitive to the temperature change compared with that of galactose and tagatose.

The dissolution enthalpy, $\Delta H_{i}$, of each hexose was evaluated from the slope of each line drawn in Fig. 1 according to Eq. (1). The estimated $\Delta H$ values are listed in Table 1. The dissolution enthalpy of any hexose decreased upon increasing the ethanol 
concentration, and the dissolution enthalpy of talose was larger than that of galactose and tagatose.

These results indicate that talose and tagatose can be separated from galactose by adding ethanol, decreasing the temperature of the reaction mixture, or by the combined use of these two means.

Acknowledgements One of the authors (D.-M. G.) gratefully acknowledges a Monbukagakusho Scholarship from the Japanese government and the Tojuro Iijima Foundation for Food Science and Technology. This study was also partly supported by JSPS KAKENHI (No. 26870296; T. K.).

\section{References}

Alves, L.A., Almeida e Silva, J.B., and Giulietti, M. (2007). Solubility of D-glucose in water and ethanol/water mixtures. J. Chem. Eng. Data, $\mathbf{5 2}$, 2166-2170.

Baer, H.H. (1962). Cyclizations of dialdehydes with nitromethane. VIII. A spontaneous epimerization in aci-nitro glycosides and its significance in the preparation of derivatives of 3-amino-3-deoxy-D-mannose, D-glucose, D-talose and D-galactose. J. Am. Chem. Soc., 84, 83-89.

Beerens, K., Desmet, T., and Soetaert, W. (2012). Enzymes for the biocatalytic production of rare sugars. J. Ind. Microbiol. Biotechnol., 39, 823-834

Flood, A.E., Addai-Mensah, J., Johns, M.R., and White, E.T. (1996). Refractive index, viscosity, density, and solubility in the system fructose
+ ethanol + water at 30,40 , and $50^{\circ}$ C. J. Chem. Eng. Data, 41, 418-421. Gao, D.-M., Kobayashi, T., and Adachi, S. (2015). Production of rare sugars from common sugars in subcritical aqueous ethanol. Food Chem., 175, 465-470.

Hudson, C.S. and Yanovsky, E. (1917). Indirect measurements of the rotatory powers of some alpha and beta forms of the sugars by means of solubility experiments. J. Am. Chem. Soc., 39, 1013-1038.

Khadem, H.S.E., Ennifar, S., and Isbell, H.S. (1989). Evidence of stable hydrogen-bonded ions during isomerization of hexoses in alkali. Carbohydr. Res., 185, 51-59.

Levin, G.V., Zehner, L.R., Saunders, J.P., and Beadle, J.R. (1995). Sugar substitutes: their energy values, bulk characteristics, and potential health benefits. Am. J. Clin. Nutr., 62, 1161s-1168s.

Lim, B.C., Kim, H.J., and Oh, D.K. (2007). A high production of D-tagatose by the addition of boric acid. Biotechnol. Progr., 23, 824-828.

Lu, Y., Levin, G.V., and Donner, T.W. (2008). Tagatose, a new antidiabetic and obesity control drug. Diabetes Obes. Metab., 10, 109-134.

Silva, A.T.C.R., Martinez, K.C.L., Brito, A.B.N., and Giulietti, M. (2010). Separation of glucose and fructose by freezing crystallization. Cryst. Res. Technol., 45, 1032-1034.

Yamauchi, T., Fukushima, K., Yanagihara, R., Osanai, S., and Yoshikawa, S. (1990). Epimerization and isomerization of various monosaccharides using metal-diamine systems. Carbohydr. Res., 204, 233-239.

Yu, S.H. and Chung, S.K. (1968). Synthesis of 2-amino-2-deoxy-D-talose and 2-amino-2-deoxy-D-galactose. Can. J. Chem., 46, 2481-2484. 